\title{
Neural response to the observable self in social anxiety disorder
}

\author{
J. Pujol $^{1 *}$, M. Giménez ${ }^{1}$, H. Ortiz ${ }^{1}$, C. Soriano-Mas ${ }^{2,3}$, M. López-Solà ${ }^{1,3}$, M. Farré ${ }^{4,5}$, J. Deus ${ }^{1,6}$, \\ E. Merlo-Pich ${ }^{7}$, B. J. Harrison ${ }^{8}$, N. Cardoner ${ }^{2,3}$, R. Navinés ${ }^{4,9}$ and R. Martín-Santos ${ }^{9,10}$ \\ ${ }^{1}$ Institut d'Alta Tecnologia-PRBB, CRC Mar, Hospital de Mar, Barcelona, Spain; ${ }^{2}$ Department of Psychiatry, Bellvitge University \\ Hospital-IDIBELL, Barcelona, Spain; ${ }^{3}$ Carlos III Health Institute, Ministry of Science and Innovation, CIBERSAM, Spain; \\ ${ }^{4}$ Human Pharmacology and Neurosciences, Hospital del Mar Research Institute (IMIM), Red RTA, Barcelona, Spain; ${ }^{5}$ Department of \\ Pharmacology, Therapeutics, and Toxicology, School of Medicine, Autonomous University of Barcelona, Spain; ${ }^{6}$ Department of Clinical and \\ Health Psychology, Autonomous University of Barcelona, Spain; ${ }^{7}$ Neuronal Targets DPU, Respiratory CEDD, GlaxoSmithKline SpA, King of \\ Prussia, PA, USA; ${ }^{8}$ Melbourne Neuropsychiatry Centre, Department of Psychiatry, The University of Melbourne, Australia; ${ }^{9}$ Clinical Institute of \\ Neuroscience, Hospital Clinic-IDIBAPS, CIBERSAM, Barcelona, Spain; ${ }^{10}$ Department of Psychiatry and Clinical Psychobiology, University of \\ Barcelona, Spain
}

Background. Distorted images of the observable self are considered crucial in the development and maintenance of social anxiety. We generated an experimental situation in which participants viewed themselves from an observer's perspective when exposed to scrutiny and evaluation by others.

Method. Twenty patients with social anxiety disorder (SAD) and 20 control subjects were assessed using functional magnetic resonance imaging (fMRI) during the public exposure of pre-recorded videos in which they were each shown performing a verbal task. The examiners acted as the audience in the experiment and rated performance. Whole-brain functional maps were computed using Statistical Parametric Mapping.

Results. Robust activation was observed in regions related to self-face recognition, emotional response and general arousal in both study groups. Patients showed significantly greater activation only in the primary visual cortex. By contrast, they showed significant deactivation or smaller activation in dorsal frontoparietal and anterior cingulate cortices relevant to the cognitive control of negative emotion. Task-related anxiety ratings revealed a pattern of negative correlation with activation in this frontoparietal/cingulate network. Importantly, the relationship between social anxiety scores and neural response showed an inverted-U function with positive correlations in the lower score range and negative correlations in the higher range.

Conclusions. Our findings suggest that exposure to scrutiny and evaluation in SAD may be associated with changes in cortical systems mediating the cognitive components of anxiety. Disorder severity seems to be relevant in shaping the neural response pattern, which is distinctively characterized by a reduced cortical response in the most severe cases.

Received 22 March 2012; Revised 9 July 2012; Accepted 13 July 2012; First published online 16 August 2012

Key words: Cognitive anxiety, emotion, fMRI, public exposure, self-recognition.

\section{Introduction}

Social anxiety disorder (SAD) is defined by a marked fear of social or performance situations in which the person is exposed to scrutiny by others (AMA, 2000). From a cognitive-behavioral viewpoint, entering a feared social situation enhances negative aspects of self-perception in SAD patients, such that distorted images of the observable self are considered crucial to the development and maintenance of social phobia

* Address for correspondence: Dr J. Pujol, Department of Magnetic Resonance, CRC Mar, Hospital del Mar, Passeig Marítim 25-29, 08003 Barcelona, Spain.

(Email: jpujol@crccorp.es)
(Clark \& Wells, 1995; Roth \& Heimberg, 2001). In essence, SAD patients do not ultimately fear the audience when exposed, but rather fear the notion of the observable self as a target for disapproval (Roth \& Heimberg, 2001).

In the current functional magnetic resonance imaging (fMRI) study, we assessed the neural response of patients to self-recognition when exposed to scrutiny and evaluation by others. Specifically, the experiment involved presenting patients with prerecorded video sequences of themselves performing a verbal task, in a session during which the examiners acted as the audience and rated performance. We anticipated that the neural response in this situation would involve robust activation of distributed brain 
regions in most subjects (with and without SAD). Indeed, simple visual recognition of one's own face is associated with the activation of a complex cortical network involving the inferior occipito-temporal cortex, inferior frontal and parietal cortices, the medial frontal gyrus and the anterior cingulate cortex (Devue \& Brédart, 2011). We also expected that a potentially tense scrutiny situation would lead to relevant engagement of emotion and arousal systems corresponding with the activation of the amygdala and the thalamus in addition to the insular, cingulate and prefrontal cortices (Barrett et al. 2007).

In SAD patients, a larger neural response would be expected if a positive correlation between generated anxiety and brain activation were assumed. Nevertheless, previous research using symptom provocation tasks suggests that this may be not the case for all brain regions. Indeed, although public speaking did produce larger activation in subcortical structures and amygdala in SAD patients, they showed a reduced cortical response compared with control subjects involving areas devoted to the evaluative processes of emotion (Tillfors et al. 2001; Lorberbaum et al. 2004). Similarly, imagining socially threatening situations was associated with reduced cortical activity in SAD patients (Kilts et al. 2006; Nakao et al. 2011). Of note, confronting patients with negative self-beliefs (Goldin et al. 2009b) and firstperson negative appraisals (Blair et al. 2011) has also been associated with reduced early activation in cortical areas related to emotion cognitive control. Finally, in an exploratory study with no control subjects, SAD patients showed decreased activity in frontal and occipital cortices during a video exposure of recorded performance similar to our task when compared with the exposition of a stranger (Van Ameringen et al. 2004).

The paradoxical absence of a generalized brain activity increases during specific symptom provocation in SAD contrasts with a more consistent response enhancement observed when relatively milder emotional stimulation paradigms have been used, such as the processing of emotional faces (Etkin \& Wager, 2007), general emotional pictures (Shah et al. 2009), phobic-related words (Schmidt et al. 2010) and emotional prosody (Quadflieg et al. 2008). In general, previous imaging data suggest that SAD patients show a pattern of increased response to milder emotional stimulation in basic emotional domains and a combination of topographically segregated increases and decreases in activity in response to more intense socially relevant provocation.

In the context of our task, we predicted that SAD patients overall would not show a generally higher response in relation to control subjects. Instead, we anticipated that patients would demonstrate a combination of activation increases in systems mediating emotional reactions (Tillfors et al. 2001), together with reduced activation of cortical areas promoting the cognitive control of anxiety (Bishop et al. 2004). Therefore, our aim was to use fMRI to investigate the extent to which our task differentially engages both components in 20 SAD patients and 20 control subjects. The continuous recording of heart rate was used as a representative measurement of the physiological response. Whole-brain functional maps were generated for task-related activation and for correlations with anxiety measurements. We also conducted an out-of-scanner behavioral experiment to further characterize the nature of the provoked anxiety and to rule out possible gaze avoidance strategies.

\section{Method}

\section{Participants}

A total of 20 patients with generalized SAD were recruited through public media advertisement (local newspapers and poster advertisements). Participants contacted the study center (Pharmacology Research Unit) by email and then a clinical researcher performed a preliminary interview by telephone. A screening visit was performed thereafter to confirm inclusion/exclusion criteria and good physical health by a complete physical examination. Inclusion criteria were: (a) out-patients with a primary psychiatric diagnosis of generalized SAD according to DSM-IVTR criteria (APA, 2000) in conjunction with the Mini International Neuropsychiatric Interview (MINI; Sheehan \& Lecrubier, 1999), (b) a Liebowitz Social Anxiety Scale (LSAS; Liebowitz, 1987) score $\geqslant 50$, and (c) participants aged between 18 and 60 years. Patients with relevant medical or neurological disorders, or other DSM-IV Axis I disorders, were not considered for inclusion. All subjects were free of any history of substance dependence or current substance abuse, and all provided a negative urine toxicity and breath alcohol screen. In addition, subjects receiving any current psychotherapy or pharmacological treatment were not included. The finally selected sample represents a notably homogeneous SAD group of generalized type (no cases showing only performancerelated SAD were included) with childhood onset of symptoms and significant distress and interference in the patient's life, but with no current treatment that could confound the study results. A group of 20 healthy volunteers matched by age, educational status and gender distribution were recruited. These control participants satisfied the same health 
Table 1. Characteristics of subject populations

\begin{tabular}{llll}
\hline & $\begin{array}{l}\text { SAD patients } \\
(n=20)\end{array}$ & $\begin{array}{l}\text { Control subjects } \\
(n=20)\end{array}$ & $p$ \\
\hline Age (years), mean (S.D.) & $24.2(5.2)$ & $24.4(5.6)$ & 0.87 \\
Gender (M/F) & $5 / 15$ & $6 / 14$ & 0.72 \\
Education: superior level, $n$ (\%) & $20(100)$ & $20(100)$ & - \\
Age of onset (years), mean (s.D.) & $9.7(5.1)$ & - & - \\
Illness duration (years), mean (s.D.) & $14.6(7.2)$ & - & - \\
LSAS score, mean (s.D.) & $80.7(16.2)$ & $11.8(8.5)$ & $<0.0005$ \\
STAI-S score, mean (S.D.) & $30.8(8.7)$ & $7.8(5.9)$ & $<0.0005$ \\
State anxiety VAS, mean (s.D.) & & & - \\
$\quad$ Before fMRI session & $54.5(19.8)$ & - & - \\
After fMRI session & $40.1(21.9)$ & - & - \\
HAMD-17 score, mean (s.D.) & $3.1(2.2)$ & - & - \\
PHQ-15 score, mean (s.D.) & $5.1(3.5)$ & - & - \\
CGI-S score, $n$ (\%) & & & - \\
$\quad$ Moderately ill & $6(30)$ & - & - \\
Markedly ill & $9(45)$ & - & - \\
Severely ill & $5(25)$ & - & - \\
\hline
\end{tabular}

SAD, Social anxiety disorder; M, male; F, female; LSAS, Liebowitz Social Anxiety Scale; STAI-S, State-Trait Anxiety Inventory - State; VAS, visual analogue scale; fMRI, functional magnetic resonance imaging; HAMD-17, 17-item Hamilton Rating Scale for Depression; PHQ-15, 15-item Patient Health Questionnaire; CGI-S, Clinical Global Impression - Severity Scale; S.D., standard deviation.

conditions and also showed negative results in the toxicity screen. All participants were right-handed.

Detailed behavioral assessments included the LSAS (Liebowitz, 1987), the State-Trait Anxiety Inventory (STAI; Spielberger, 1983) and 0-100-mm visual analogue scales (VAS) as ratings of state anxiety, the Hamilton Rating Scale for Depression (HAMD; Hamilton, 1960), the Patient Health Questionnaire (PHQ; Spitzer et al. 1999) and the Clinical Global Impression - Severity Scale (CGI-S; Guy, 1976). The characteristics of both study groups are described in Table 1. Written informed consent was obtained from all participants. The study was approved by the local ethics committee (CEIC-IMAS, Barcelona) and was in compliance with the Declaration of Helsinki.

\section{fMRI experiment}

\section{Experimental task}

Before scanning, participants' verbal task performances were video-recorded privately in a laboratory setting. They underwent a memory task adapted from the Wechsler Memory Scale-Revised (Wechsler, 1987), which involves a single presentation of verbal narratives to be recalled immediately. Subjects were asked to listen to and repeat aloud three stories. During each repetition, video sequences of 30-s duration were recorded at a short distance. Public exposure to the video segments occurred only during the fMRI session when both the subject and the research team viewed the scenes. Participants were informed that a clinical psychologist would evaluate their memory performance during the imaging session according to formal guidelines. As a control condition, equivalent video segments featuring unknown 'other' subjects responding to the same stories were used. These 'others' were matched in terms of age and gender to each individual study participant. We maintained a stable background (neutral white color) and framing (fixed general short plane) in all videos, and avoided possible distracters such as loud colors for clothes, hairstyle or accessories (large pendants or earrings).

In total, the fMRI experiment consisted of six alternating 30-s blocks of the control (other) and experimental (self) conditions. It is important to note that the experiment required no actual performance during scanning. In this way, the neural response shows no influence from actions and mental operations related to performance. This may facilitate the interpretation of results and provide data less affected by head motion artifacts. Total recall execution scores during the videos were not collected, as the task finished once a 30-s video was successfully recorded. To assess 
the quality of execution, we considered relevant, that participants were talking during most (80\%) of the recording periods. That is, periods of silence during recording never exceeded $20 \%$ of the time. Experimental and baseline conditions were also equated in this performance parameter.

\section{Physiological data acquisition}

Heart rate was monitored continuously during fMRI scans using a photoplethysmograph placed on the left index finger (Model 4500MRI, Invivo Corp., USA). The device provided an output signal for each arterial pulsation that was registered using software developed in-house on the Labview 8.0 platform (National Instruments Corp., USA). Scanner trigger pulses were also registered to allow accurate synchronization of physiological signals with the fMRI data. Data were analyzed and plotted using procedures similar to those reported in a previous study (Caseras et al. 2010). The beat-to-beat interval was calculated and the inverse of each interval was designated as the beat-tobeat heart rate. The evoked response to the experimental (self) condition was calculated as the heart rate increase in relation to the preceding control (other) block. Group mean heart rate was also calculated for each block.

\section{Image acquisition and preprocessing}

We used a 1.5-T Signa Excite system (General Electric, USA) equipped with an eight-channel phased-array head coil and single-shot echo-planar imaging (EPI) software. Functional sequences consisted of gradient recalled acquisition in the steady state [repetition time $(\mathrm{TR})=2000 \mathrm{~ms}$; echo time $(\mathrm{TE})=50 \mathrm{~ms}$; pulse angle $=90^{\circ}$ ] within a field of view of $24 \mathrm{~cm}$, with a $64 \times 64$-pixel matrix and a slice thickness of $4 \mathrm{~mm}$ (interslice gap $=1.5 \mathrm{~mm}$ ). Twenty-two interleaved slices, parallel to the anterior-posterior commissure line, were acquired to cover the whole brain. The first four (additional) images in each run were discarded to allow the magnetization to reach equilibrium. The fMRI task was generated and timing was controlled by Presentation ${ }^{\mathrm{TM}}$ software (Neurobehavioral Systems Inc., USA; www.neurobs.com/) and presented to subjects using MRI-compatible high-resolution goggles and an audio headset (VisuaStim Digital System, Resonance Technology Inc., USA). fMRI data were preprocessed and analyzed using the Statistical Parametric Mapping 5 (SPM5) package, Wellcome Department of Imaging Neuroscience (www.fil.ion. ucl.ac.uk/spm/), running on Matlab 7 (The Mathworks Inc., USA). Functional images were realigned (motion corrected) and spatially warped into the standardized SPM template space. A full-width at half-maximum 8-mm Gaussian kernel was used to smooth the functional images.

\section{fMRI statistical analysis}

Single-subject (first-level) SPM contrast images were estimated comparing the 'self' condition with the 'other' condition. For these analyses, the fMRI signal response at each voxel was modeled using the SPM canonical hemodynamic response function. The resulting first-level contrast images were then carried forward to subsequent second-level random-effects (group) analyses. One-sample $t$-statistic maps were calculated to obtain task-related activations and deactivations, and two-sample $t$ tests were performed to map between-group differences. Voxel-wise analysis in SPM5 was also performed to map the correlation between brain activation and both task-related anxiety ratings and LSAS scores. The task-related anxiety ratings were (i) 'anxiety before minus anxiety after scanning', as a representative measurement of anticipatory anxiety obtained in the fMRI session day, and (ii) 'anxiety during 'self' condition minus anxiety during 'other' condition', as a task-evoked anxiety measurement obtained in the out-of-scanner experiment. A threshold $p_{\text {FDR }}<0.05$ whole-brain corrected was used in this study. In one-sample $t$-test maps, only activations surviving this conservative threshold are reported. For between-group comparisons and correlation maps, changes involving a minimum cluster extension of 15 voxels at $p<0.001$ uncorrected were also reported, which may provide an optimal balance between type I and type II errors (Lieberman \& Cunningham, 2009). $p<0.01$ is used for display purposes.

\section{Out-of-scanner behavioral experiment}

Because of the complexity of the fMRI session (Giménez et al. 2012), the assessment of task behavioral effects was completed in a separate experiment that included eye-tracking records to rule out possible visual avoidance strategies in SAD patients during symptom provocation (Horley et al. 2003; Pujol et al. 2009) (see online Supplementary Method).

\section{Results}

\section{fMRI experiment}

\section{Brain activation}

The neural response to the 'self' versus 'other' condition produced robust activation in regions involved in self-face recognition (extrastriate visual cortex, right inferior frontal gyrus and medial frontal gyrus) and 


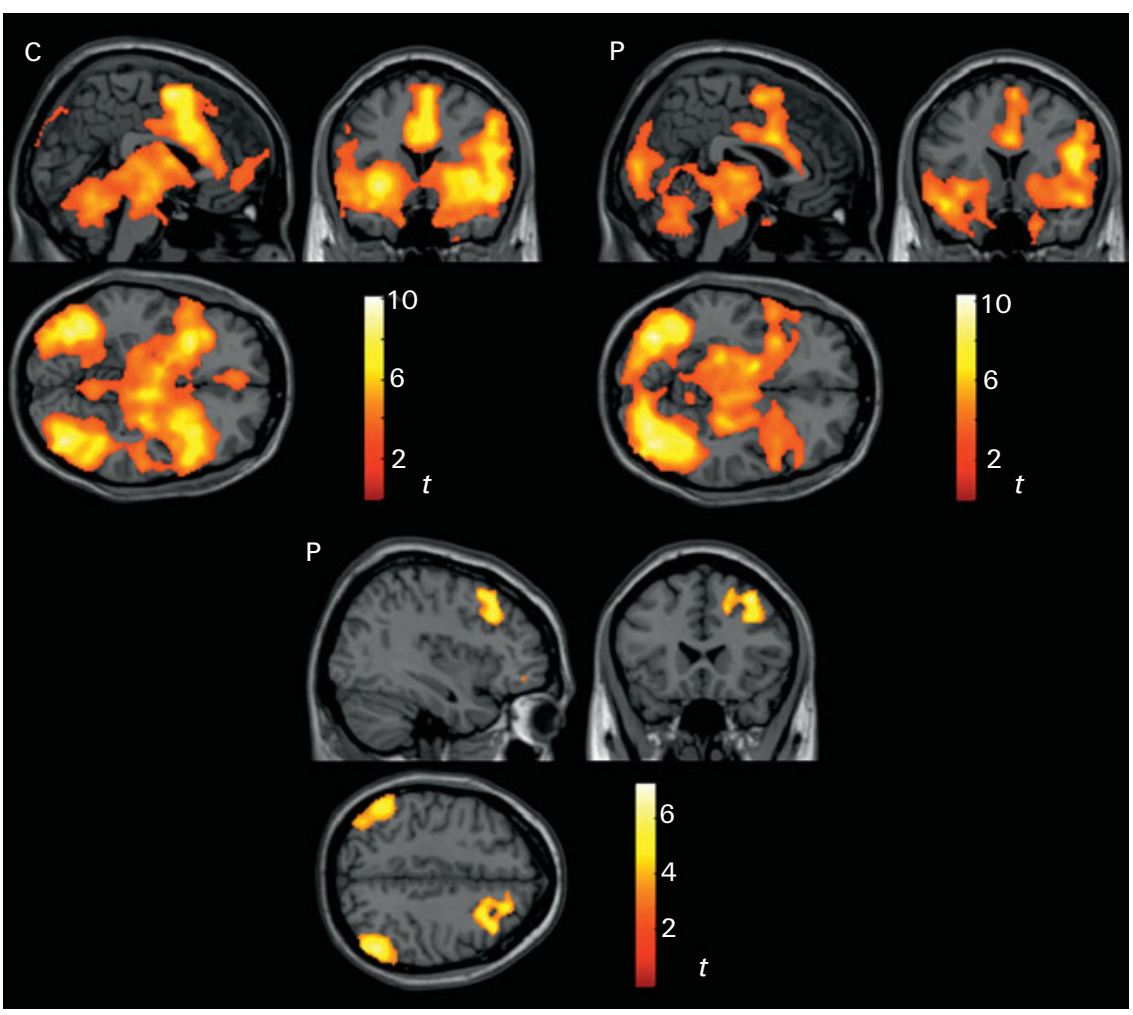

Fig. 1. Neural response to the task. Activation one-sample $t$ maps for control subjects (C) and social anxiety disorder (SAD) patients (P) (top) and deactivation maps for SAD patients (bottom). Deactivation in control subjects did not reach the significance level. The right hemisphere corresponds to the right side of the coronal views and the bottom side of the axial views.

emotional response/general arousal (bilateral anterior insula, anterior cingulate cortex, bilateral amygdala, upper brainstem, thalamus, basal ganglia and cerebellum) in both SAD patients and control subjects. SAD patients showed additional activation in the primary visual cortex but the activation in midline regions was less extensive (Fig. 1, and online Supplementary Table S1). Significant deactivation during the 'self' condition was observed only in the patient group in the dorsal prefrontal and parietal neocortex. The direct between-group comparison showed no false-discovery rate (FDR)-corrected differences. Nevertheless, increased activation in the primary visual cortex, reduced activation in the medial frontal gyrus and the anterior cingulate cortex, and more pronounced deactivation in the dorsolateral prefrontal cortex were identified in SAD patients compared to control subjects at $p<0.001$ uncorrected (cluster extension $>15$ voxels) (Fig. 2, Table S1).

\section{Correlation of brain activation with task-related anxiety ratings}

SAD patients showed a notable amount of anxiety before the fMRI assessment (Table 1). Using 'anxiety before minus anxiety after scanning' as a measurement of anticipatory anxiety, we observed a negative correlation with cortical activation within the patient group $(p<0.001$ uncorrected, cluster $>15$ voxels) (Fig. $3 a$, Table S2). Anxiety ratings obtained in the separate behavioral experiment similarly showed negative correlations with brain activation, in this case combining patients and controls in the measurement of anxiety during 'self' condition minus anxiety during 'other' condition (Fig. 3b, Table S2). Of note, in both analyses the areas with negative correlations implicated the frontoparietal/cingulate network that showed significant deactivation or reduced activation during the task in patients.

\section{Correlation of brain activation with social anxiety scores}

The correlation between LSAS scores and the observed brain response showed a clear tendency to differ between groups. LSAS correlated positively with brain activation in control subjects in regions where SAD patients showed negative correlations. The map of the interaction between groups for the correlation of LSAS with brain response summarizes this effect (Fig. 4, Table S3). Regions showing an interaction effect included the mesencephalic tegmentum, the thalamus, hypothalamus, ventral striatum, medial and 


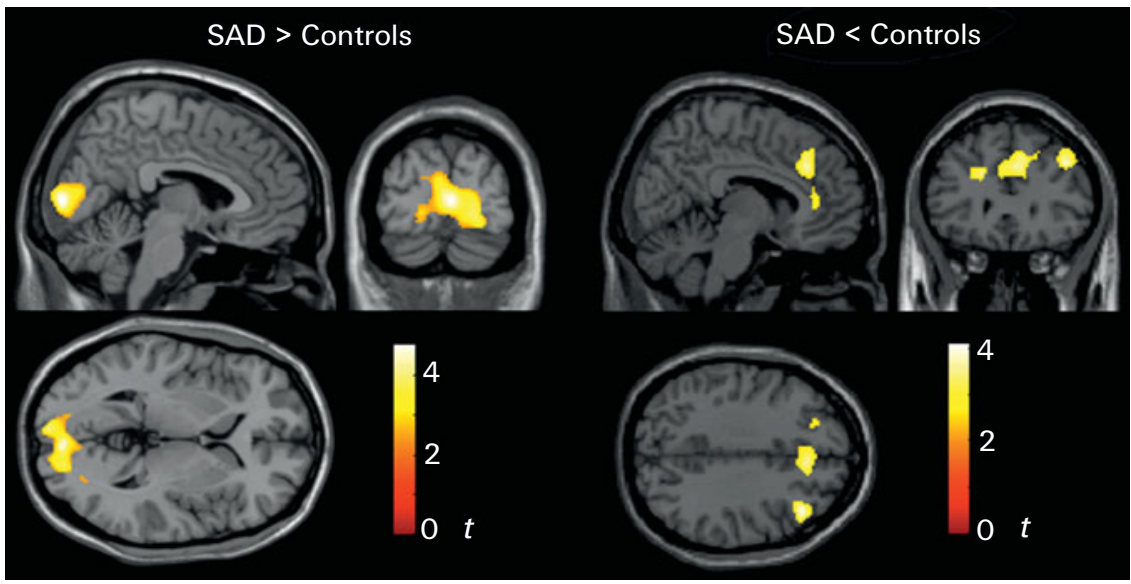

Fig. 2. Between-group differences in task responses. The right hemisphere corresponds to the right side of the coronal views and the bottom side of the axial views.

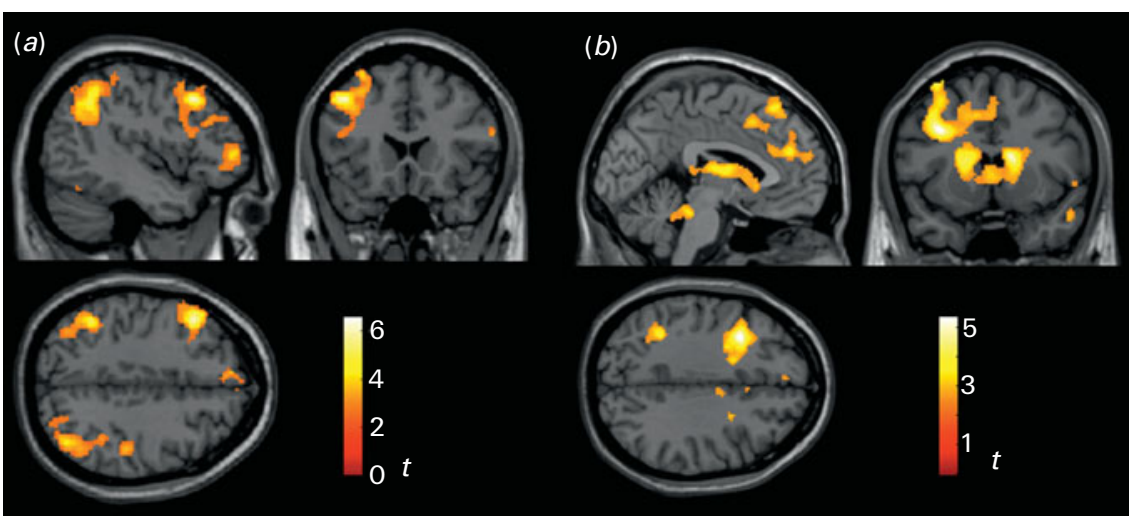

Fig. 3. Correlation of brain activation with task-related anxiety ratings. (a) Images show the correlation of brain activation with anticipatory anxiety before functional magnetic resonance imaging (fMRI) in 20 patients. (b) Images show the correlation of brain activation with anxiety provoked by the task in the separate experiment combining patients and control subjects (total $n=30$ ). The right hemisphere corresponds to the right side of the coronal views and the bottom side of the axial views.

posterior orbitofrontal cortex, occipitotemporal areas and the right dorsal (medial and lateral) frontal cortex. The interaction effect is illustrated with a plot including both controls and patients (Fig. 4). For this plot, the relationship between LSAS and brain activation was best explained by a quadratic or inverted U-shaped function $\left(r=0.75, r^{2}=0.56, p<0.00001\right)$.

The neural response to symptom provocation showed a wide activation range in SAD patients as a function of disorder severity (Fig. 4). In a post-hoc analysis, patients with the lowest LSAS scores $(n=10)$ and those with the highest scores $(n=10)$ were separately compared to the control group $(n=20)$ (online supplementary Fig. S1). The lower severity group showed greater activation than controls in posterior brain areas including the primary visual cortex, fusiform gyrus, posterior mesencephalon and the parahippocampal gyrus at its junction with the amygdala: peak activation $t=6.32$ and $p<0.0005$ at Montreal
Neurological Institute (MNI) coordinates $x=20$, $y=-90, \quad z=-8 \mathrm{~mm}$. The higher severity group showed reduced activation compared to controls in anterior brain areas including the anterior cingulate cortex, medial frontal cortex and orbitofrontal cortex (peak activation $t=4.9$ and $p<0.0005$ at $\mathrm{x}=6, \mathrm{y}=36$, $\mathrm{z}=4)$ and in the thalamus $(t=3.6$ and $p=0.001$ at $x=10, y=-12, z=16)$.

\section{Physiological monitoring}

Recorded heart data were optimal ( $>95 \%$ of beats registered) for all but three participants who were subsequently excluded from the analysis (one control subject and two patients). SAD patients showed a tendency to lower heart rate during each paradigm phase; in terms of the whole experiment, control subjects showed an average heart rate of $78.8 \pm 10.3$ beats/min and SAD patients $72.2 \pm 9.5$ beats $/ \mathrm{min}$ 


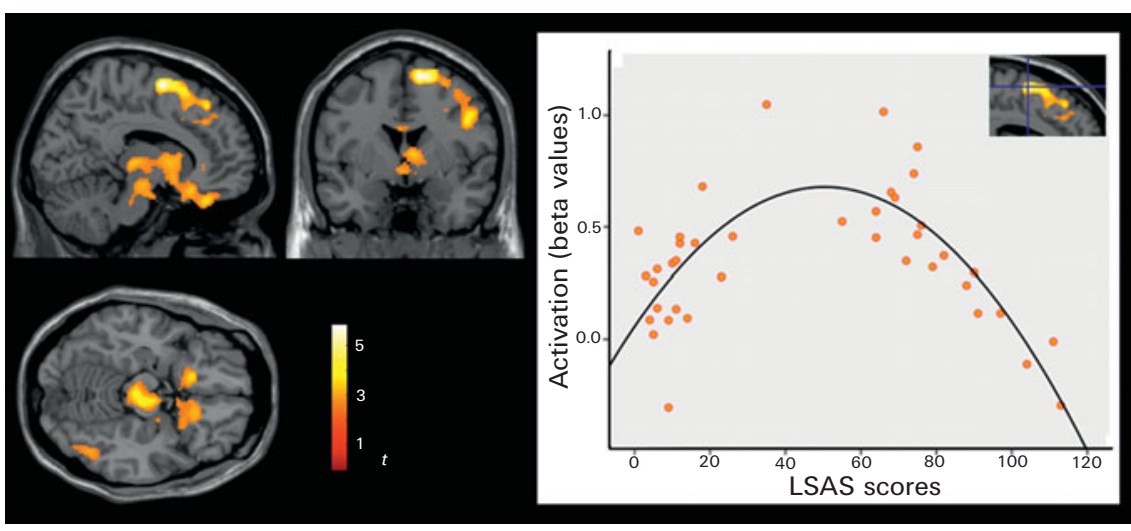

Fig. 4. Correlation of brain activation with Liebowitz Social Anxiety Scale (LSAS) scores. The map corresponds to the interaction between groups and displays voxels showing a positive correlation significantly greater in control subjects than in patients (or a negative correlation significantly greater in patients than in controls). The right hemisphere corresponds to the right side of the coronal view and the bottom side of the axial view. The plot illustrates the interaction effect. A quadratic function or an inverted-U function provided the best explanation for the relationship.

$(t=2.0$ and $p=0.051)$. The response to the 'self' condition was characterized by an initial increase in heart rate in control subjects (Fig. S2). This physiological reaction, however, was mostly absent in SAD patients. The mean initial (5-s window) heart rate increase across the three 'self' condition blocks was $1.9 \pm 2.6$ beats $/ \mathrm{min}$ in control subjects and $0.4 \pm 1.1$ beats $/ \mathrm{min}$ in SAD patients $(t=2.4$ and $p=0.025)$.

\section{Out-of-scanner behavioral experiment}

This experiment confirmed that the exposure to their own pre-videoed performance generated a significant amount of anxiety in SAD patients (Supplementary Results and Fig. S3).

\section{Discussion}

To investigate a crucial process in the development and maintenance of social anxiety, we generated an experimental situation in which participants were able to see themselves as if viewed from an observer's perspective during exposure to scrutiny and evaluation by others. SAD patients were highly sensitive to the situation, as reflected by their behavioral ratings. However, both groups demonstrated robust activation in brain regions related to self-face recognition, emotion and general arousal. The patients showed greater activation than the controls only in primary visual areas. By contrast, the patients showed deactivation or smaller activation in dorsal frontoparietal and anterior cingulate cortices. Task-related anxiety ratings revealed a pattern of negative correlation with activation in this frontoparietal/cingulate network. Social anxiety scores were positively correlated with brain activation in control subjects and negatively in patients, a pattern that was best explained by an inverted-U function.

At first sight, it may seem paradoxical that a situation generating relevant stress in SAD patients was not associated with a generally larger neural response compared to controls. Nevertheless, previous provocation studies in SAD have reported both activity increases and decreases (Tillfors et al. 2001; Lorberbaum et al. 2004; Van Ameringen et al. 2004; Kilts et al. 2006; Goldin et al. 2009 b; Blair et al. 2010, 2011; Nakao et al. 2011), which would suggest that the brain response to such situations may be complex and dependent on a variety of factors, including specific task characteristics, clinical phenotype and disorder severity. The response to public speaking and to its anticipation was associated with significant amygdala activation in previous reports (Tillfors et al. 2001; Lorberbaum et al. 2004). Our task did indeed cause significant amygdala activation in both study groups, but patients did not exhibit larger activation than controls. Therefore, our experimental situation does not seem to generate a salient response in the basic emotional response systems in SAD, as has been shown in other contexts (Tillfors et al. 2001; Lorberbaum et al. 2004; Blair et al. 2010). The nature of our task ('indirect exposure', where the participants are watching themselves after the fact) may favor more engagement of cognitive processes, including post-hoc analysis of their own performance, and less limbic/paralimbic participation, as opposed to tasks involving live exposure to public speaking (Tillfors et al. 2001). In contrast with the lack of overt limbic hyper-response, it is relevant to note that the largest between-group difference was the increase in visual cortex activation in SAD. This 
finding is in concordance with previous studies postulating an important role of the visual cortex in emotional arousal at the perception level (Sabatinelli et al. 2007, 2012; McTeague et al. 2011).

In our assessment, symptom provocation was not associated with increased psychophysiological arousal, but instead SAD patients showed reduced heart rate responses. Several independent research groups have reported an absence of a significant increase in heart rate and other physiological measures between SAD patients and controls in response to threatening faces (see Staugaard, 2010 for a review), or during stressful speaking tasks (Tillfors et al. 2001; Edelmann \& Baker, 2002; Mauss et al. 2004; Heiser et al. 2009; Schmitz et al. 2011). Physiological arousal, however, may be relevant in particular patient subgroups. For example, significant heart rate acceleration during speech presentation was observed in social phobia patients who complained of blushing (Gerlach et al. 2001), elderly socially anxious women (Grossman et al. 2001), social phobics without associated avoidance personality disorder (Hofmann et al. 1995), and selective public speaking phobics (as opposed to generalized social phobia) (Heimberg et al. 1990; Levin et al. 1993). Nevertheless, it is important to emphasize that differences between patients and control subjects have been more consistent for the subjective perception of physiological reactions than for the reactions themselves (Gerlach et al. 2001, 2004; Grossman et al. 2001 ; Edelmann \& Baker, 2002; Mauss et al. 2004; Wild et al. 2008), which supports cognitive theories of social anxiety emphasizing negative interpretation of bodily sensations as part of the anxiety response (Edelmann \& Baker, 2002; Mauss et al. 2004; Wild et al. 2008).

Although anxiety may potentially be associated with either excessive emotional (limbic) reactivity or its insufficient cognitive (cortical) control (Bishop et al. 2004), the failure of emotion regulation is thought to be a key feature of SAD (Kilts et al. 2006; Goldin et al. $2009 b$ ). The effect of cognitive-linguistic regulation of emotional reactivity to social threat (Goldin et al. 2009a) and to negative self-beliefs (Goldin et al. 2009b) was explicitly assessed in two different experiments. In both, the authors reported reduced activation in brain areas related to attention and cognitive control of emotion in SAD patients compared to healthy controls. This effect may be not generalized to all anxiety types, as individuals prone to general anxiety (defined by Spielberger STAI scores) seem instead to engage prefrontal and anterior cingulate regions more than healthy controls during the cognitive modulation of negative emotion (Campbell-Sills et al. 2011). We observed normal subcortical and limbic response with reduced activation in cortical areas, a pattern that is compatible with a failure in the cognitive control of anxiety. Importantly, we found decreased activation in SAD patients specifically in the core of both the prefrontal and the cingulate systems, which jointly mediate the cognitive control of negative emotion through both attention strategies and cognitively changing the meaning of emotionally evocative stimuli (Ochsner \& Gross, 2005; Phillips et al. 2008).

Research in healthy individuals has demonstrated the association of higher anxiety levels with less activity specifically in prefrontal and rostral anterior cingulate cortices in response to threat-related stimulation (Bishop et al. 2004). In our study, both anticipatory anxiety and anxiety during provocation showed a negative correlation with activation in prefrontal and dorsal anterior cingulate cortices, which would further support the notion that cognitive anxiety, as opposed to somatic anxiety, is relevant in SAD when fear of the observable self is provoked. Significant correlation with anxiety ratings was also observed in the control group, which suggests that social anxiety may be associated with a weaker recruitment of top-down control mechanisms across the entire range of this dimensional disorder. Other studies in SAD, however, have found a positive relationship between provoked anxiety and brain response in structures more closely related to emotional reactivity, such as the amygdala (Tillfors et al. 2001) and the most ventral part of the prefrontal cortex (Kilts et al. 2006).

A classical concept relates anxiety to better performance accuracy at moderate levels and to worsening performance when anxiety intensity increases (the inverted ' $U$ ' law introduced by Yerkes \& Dodson in 1908). In our study, the neural response to symptom provocation showed similar dynamics. We found a positive correlation between LSAS severity and brain activation in the low score range and a negative correlation with higher scores. As a result, the neural response in the most severe patients was markedly decreased. Non-linear associations with an inverted U-shape were observed during anticipatory anxiety to pain for the anterior cingulate cortex (Straube et al. 2009). In a study assessing a large sample of patients with lifetime anxiety disorder, anxious arousal showed a similar non-linear association with cortisol awakening response (Wardenaar et al. 2011), which is of interest given that our analysis also implicated the hypothalamus. An earlier study in healthy volunteers observed the inverted U-shaped relationship between anxiety and both cerebral blood flow and cerebral metabolic rates for glucose (Gur et al. 1987). The authors proposed a fall in brain activity during high stress as a physiological explanation of the inverted-U relationship between anxiety and performance. It may be of interest in future studies to investigate whether 
the inverted-U dynamics accounts for the experience of 'mental block' frequently reported in highly anxious subjects during performance.

As a study limitation, we should point out that the assessment of subjective anxiety during the fMRI session only involved anticipatory anxiety measurements, whereas a more comprehensive assessment of anxiety provoked by the task was assessed in a separate session including only a part of the original sample. Relevant variations in the clinical status of these patients between the fMRI session and the behavioral testing may be expected. Treatment, for example, could have a direct effect on task performance. We verified, however, that substantial treatment variations did not occur during this period in this subsample: only one patient received pharmacological treatment $(0.5 \mathrm{mg}$ citalopram a day) and no one received psychotherapy.

Another relevant study limitation relates to the potential effect on brain activity of the baseline condition used (viewing a stranger). This control condition was also perceived as more anxious in SAD patients (Table S4) and this is logical because SAD patients typically fear social interaction. Potential brain activations associated with increased stress during baseline in SAD could restrict the detectable activation range related to the experimental condition. In this context, the results need to be specifically interpreted as the added effect of viewing oneself compared with viewing a stranger, which was our primary aim within the framework of cognitive-behavioral models proposing the enhancement of negative aspects of self-perception in SAD patients.

It is also important to note that, although the reported findings are fairly consistent in terms of functional anatomy, group differences in brain activation and correlations were only identified at a lenient threshold. Future research will help to confirm the strength of such associations. Similarly, a more comprehensive assessment including the entire range of SAD scores, with more cases covering intermediate scores, may be useful for defining the inverted-U correlation in more detail.

In conclusion, self-recognition when exposed to scrutiny and evaluation by others generated a robust neural response in regions relevant to emotional reactivity, but group differences for the activations were not evident in these domains. Social anxiety was more specifically associated with changes in the dorsal frontal and cingulate systems mediating top-down emotion regulation, which may emphasize the relevance of the cognitive component of anxiety in the disorder. Disorder severity was important in shaping the neural response pattern, which was characterized by a clear cortical reduction in the most severe cases.
This observation may help to explain some of the divergent results that can be found in research studies of SAD.

\section{Supplementary material}

For supplementary material accompanying this paper visit http:/ /dx.doi.org/10.1017/S0033291712001857.

\section{Acknowledgments}

This study was supported in part by the Carlos III Health Institute (PI05/2565 and PI09/01331) and the Spanish Ministerio de Ciencia e Innovación (I+D+I SAF2010-19434). Dr Deus is a member of the Research Group SGR1450 and Drs Navinés and Martín-Santos of the Research Group SGR1435 from the Agency of University and Research Funding Management of the Catalonia Government. Dr López-Solà is supported by an FPU grant AP2005-0408 from the Ministry of Education of Spain. Dr Soriano-Mas is funded by a Miguel Servet contract from the Carlos III Health Institute (CP10/00604). Dr Harrison is supported by a National Health and Medical Research Council of Australia (NHMRC) Clinical Career Development Award (ID 628509).

\section{Declaration of Interest}

None.

\section{References}

APA (2000). Diagnostic and Statistical Manual of Mental Disorders, 4th edn, text revision (DSM-IV-TR). American Psychiatric Association: Washington, DC.

Barrett LF, Mesquita B, Ochsner KN, Gross JJ (2007). The experience of emotion. Annual Review of Psychology 58, 373-403.

Bishop S, Duncan J, Brett M, Lawrence AD (2004). Prefrontal cortical function and anxiety: controlling attention to threat-related stimuli. Nature Neuroscience 7, 184-188.

Blair KS, Geraci M, Hollon N, Otero M, DeVido J, Majestic C, Jacobs M, Blair RJ, Pine DS (2010). Social norm processing in adult social phobia: atypically increased ventromedial frontal cortex responsiveness to unintentional (embarrassing) transgressions. American Journal of Psychiatry 167, 1526-1532.

Blair KS, Geraci M, Otero M, Majestic C, Odenheimer S, Jacobs M, Blair RJ, Pine DS (2011). Atypical modulation of medial prefrontal cortex to self-referential comments in generalized social phobia. Psychiatry Research 193, 38-45.

Campbell-Sills L, Simmons AN, Lovero KL, Rochlin AA, Paulus MP, Stein MB (2011). Functioning of neural systems supporting emotion regulation in anxiety-prone individuals. NeuroImage 54, 689-696. 
Caseras X, Mataix-Cols D, Trasovares MV, López-Solà M, Ortriz H, Pujol J, Soriano-Mas C, Giampietro V, Brammer MJ, Torrubia R (2010). Dynamics of brain responses to phobic-related stimulation in specific phobia subtypes. European Journal of Neuroscience 32, 1414-1422.

Clark DM, Wells A (1995). A cognitive model of social phobia. In Social Phobia: Diagnosis, Assessment, and Treatment (ed. R. G. Heimberg, M. R. Liebowitz, D. A. Hope and F. R. Schneier), pp. 69-93. Guilford Press: New York.

Devue C, Brédart S (2011). The neural correlates of visual self-recognition. Consciousness and Cognition 20, 40-51.

Edelmann RJ, Baker SR (2002). Self-reported and actual physiological responses in social phobia. British Journal of Clinical Psychology 41, 1-14.

Etkin A, Wager TD (2007). Functional neuroimaging of anxiety: a meta-analysis of emotional processing in PTSD, social anxiety disorder, and specific phobia. American Journal of Psychiatry 164, 1476-1488.

Gerlach AL, Mourlane D, Rist F (2004). Public and private heart rate feedback in social phobia: a manipulation of anxiety visibility. Cognitive Behaviour Therapy 33, 36-45.

Gerlach AL, Wilhelm FH, Gruber K, Roth WT (2001). Blushing and physiological arousability in social phobia. Journal of Abnormal Psychology 110, 247-258.

Giménez M, Pujol J, Ortiz H, Soriano-Mas C, López-Solà M, Farré M, Deus J, Merlo-Pich ME, Martín-Santos R (2012). Altered brain functional connectivity in relation to perception of scrutiny in social anxiety disorder. Psychiatry Research. Published online: 18 July 2012. doi:10.1016/ j.pscychresns.2011.10.008.

Goldin PR, Manber T, Hakimi S, Canli T, Gross JJ (2009a). Neural bases of social anxiety disorder: emotional reactivity and cognitive regulation during social and physical threat. Archives of General Psychiatry 66, 170-180.

Goldin PR, Manber-Ball T, Werner K, Heimberg R, Gross JJ (2009b). Neural mechanisms of cognitive reappraisal of negative self-beliefs in social anxiety disorder. Biological Psychiatry 66, 1091-1099.

Grossman P, Wilhelm FH, Kawachi I, Sparrow D (2001). Gender differences in psychophysiological responses to speech stress among older social phobics: congruence and incongruence between self-evaluative and cardiovascular reactions. Psychosomatic Medicine 63, 765-777.

Gur RC, Gur RE, Resnick SM, Skolnick BE, Alavi A, Reivich M (1987). The effect of anxiety on cortical cerebral blood flow and metabolism. Journal of Cerebral Blood Flow and Metabolism 7, 173-177.

Guy W (ed) (1976). Clinical Global Impressions (CGI) Scale. ECDEU Assessment Manual for Psychopharmacology. US Department of Health, Education, and Welfare: Rockville, MD.

Hamilton M (1960). A rating scale for depression. Journal of Neurology, Neurosurgery and Psychiatry 23, 56-62.

Heimberg RG, Hope DA, Dodge CS, Becker RE (1990). DSM-III-R subtypes of social phobia. Comparison of generalized social phobics and public speaking phobics. Journal of Nervous and Mental Disease 178, 172-179.
Heiser NA, Turner SM, Beidel DC, Roberson-Nay R (2009). Differentiating social phobia from shyness. Journal of Anxiety Disorders 23, 469-476.

Hofmann SG, Newman MG, Ehlers A, Roth WT (1995). Psychophysiological differences between subgroups of social phobia. Journal of Abnormal Psychology 104, 224-231.

Horley K, Williams LM, Gonsalvez C, Gordon E (2003). Social phobics do not see eye to eye: a visual scanpath study of emotional expression processing. Journal of Anxiety Disorders 17, 33-44.

Kilts CD, Kelsey JE, Knight B, Ely TD, Bowman FD, Gross RE, Selvig A, Gordon A, Newport DJ, Nemeroff CB (2006). The neural correlates of social anxiety disorder and response to pharmacotherapy. Neuropsychopharmacology 31, 2243-2253.

Levin AP, Saoud JB, Strauman T, Gorman JM, Fyer AJ, Crawford R, Liebowitz MR (1993). Responses of 'generalized' and 'discrete' social phobics during public speaking. Journal of Anxiety Disorders 7, 207-221.

Lieberman MD, Cunningham WA (2009). Type I and type II error concerns in $\mathrm{fMRI}$ research: re-balancing the scale. Social Cognitive and Affective Neuroscience 4, 423-428.

Liebowitz MR (1987). Social phobia. Modern Problems of Pharmacopsychiatry 22, 141-173.

Lorberbaum JP, Kose S, Johnson MR, Arana GW, Sullivan LK, Hamner MB, Ballenger JC, Lydiard RB, Brodrick PS, Bohning DE, George MS (2004). Neural correlates of speech anticipatory anxiety in generalized social phobia. NeuroReport 5, 2701-2705.

Mauss IB, Wilhelm FH, Gross JJ (2004). Is there less to social anxiety than meets the eye? Emotion experience, expression, and bodily responding. Cognition and Emotion 18, 631-662.

McTeague LM, Shumen JR, Wieser MJ, Lang PJ, Keil A (2011). Social vision: sustained perceptual enhancement of affective facial cues in social anxiety. NeuroImage 54, $1615-1624$.

Nakao T, Sanematsu H, Yoshiura T, Togao O, Murayama K, Tomita M, Masuda Y, Kanba S (2011). fMRI of patients with social anxiety disorder during a social situation task. Neuroscience Research 69, 67-72.

Ochsner KN, Gross JJ (2005). The cognitive control of emotion. Trends in Cognitive Science 9, 242-249.

Phillips ML, Ladouceur CD, Drevets WC (2008). A neural model of voluntary and automatic emotion regulation: implications for understanding the pathophysiology and neurodevelopment of bipolar disorder. Molecular Psychiatry 13, 829-857.

Pujol J, Harrison BJ, Ortiz H, Deus J, Soriano-Mas C, López-Solà M, Yücel M, Perich X, Cardoner N (2009). Influence of the fusiform gyrus on amygdala response to emotional faces in the non-clinical range of social anxiety. Psychological Medicine 39, 1177-1187.

Quadflieg S, Mohr A, Mentzel HJ, Miltner WH, Straube T (2008). Modulation of the neural network involved in the processing of anger prosody: the role of task-relevance and social phobia. Biological Psychology 78, 129-137.

Roth DA, Heimberg RG (2001). Cognitive-behavioral models of social anxiety disorder. Psychiatric Clinics of North America 24, 753-771. 
Sabatinelli D, Keil A, Frank DW, Lang PJ (2012). Emotional perception: correspondence of early and late event-related potentials with cortical and subcortical functional MRI. Biological Psychiatry. Published online: 26 April 2012. doi:10.1016/j.biopsycho.2012.04.005.

Sabatinelli D, Lang PJ, Keil A, Bradley MM (2007). Emotional perception: correlation of functional MRI and event-related potentials. Cerebral Cortex 17, 1084-1091.

Schmidt S, Mohr A, Miltner WH, Straube T (2010). Taskdependent neural correlates of the processing of verbal threat-related stimuli in social phobia. Biological Psychology 84, 304-312.

Schmitz J, Krämer M, Tuschen-Caffier B, Heinrichs N, Blechert J (2011). Restricted autonomic flexibility in children with social phobia. Journal of Child Psychology and Psychiatry 52, 1203-1211.

Shah SG, Klumpp H, Angstadt M, Nathan PJ, Phan KL (2009). Amygdala and insula response to emotional images in patients with generalized social anxiety disorder. Journal of Psychiatry and Neuroscience 34, 296-302.

Sheehan DV, Lecrubier Y (1999). M.I.N.I. Mini International Neuropsychiatric Interview. Spanish version 5.0.0 edited by L. Ferrendo, J. M. Bobes and J. Gilbert. Instituto IAP: Madrid.

Spielberger C (1983). Manual for the State-Trait Anxiety Inventory (Form Y). Consulting Psychologists: Palo Alto, CA.

Spitzer RL, Kroenke K, Williams JB (1999). Validation and utility of a self-report version of PRIME-MD: the
PHQ primary care study. Journal of the American Medical Association 282, 1737-1744.

Staugaard SR (2010). Threatening faces and social anxiety: a literature review. Clinical Psychology Review 30, 669-690.

Straube T, Schmidt S, Weiss T, Mentzel HJ, Miltner WH (2009). Dynamic activation of the anterior cingulate cortex during anticipatory anxiety. NeuroImage 44, 975-981.

Tillfors M, Furmark T, Marteinsdottir I, Fischer H, Pissiota A, Långström B, Fredrikson M (2001). Cerebral blood flow in subjects with social phobia during stressful speaking tasks: a PET study. American Journal of Psychiatry 158, 1220-1226.

Van Ameringen M, Mancini C, Szechtman H, Nahmias C, Oakman JM, Hall GB, Pipe B, Farvolden P (2004). A PET provocation study of generalized social phobia. Psychiatry Research 132, 13-18.

Wardenaar KJ, Vreeburg SA, van Veen T, Giltay EJ, Veen G, Penninx BW, Zitman FG (2011). Dimensions of depression and anxiety and the hypothalamo-pituitaryadrenal axis. Biological Psychiatry 69, 366-373.

Wechsler D (1987). The Wechsler Memory Scale - Revised. Psychological Corporation: San Antonio, TX.

Wild J, Clark DM, Ehlers A, McManus F (2008). Perception of arousal in social anxiety: effects of false feedback during a social interaction. Journal of Behavior Therapy and Experimental Psychiatry 39, 102-116.

Yerkes RM, Dodson JD (1908). The relation of strength of stimulus to rapidity of habit-formation. Journal of Comparative Neurology and Psychology 18, 459-482. 